\title{
Nanomechanics of the Formation of DNA Self-Assembled Monolayers and Hybridization on Microcantilevers
}

\author{
M. Álvarez, ${ }^{\dagger}$ L. G. Carrascosa, ${ }^{\dagger}$ M. Moreno, ${ }^{\ddagger}$ A. Calle, ${ }^{\dagger}$ Á. Zaballos, ${ }^{\ddagger}$ \\ L. M. Lechuga, ${ }^{\dagger}$ C. Martínez-A, ${ }^{\ddagger}$ and J . Tamayo*,† \\ Biosensors Group, Instituto de Microel ectrónica de Madrid, I MM-CSIC, \\ I saac Newton 8 (PTM), 28760 Madrid, Spain, and Centro Nacional de Biotecnología, \\ DIO-CNB-CSIC, Cantoblanco, 28049 Madrid, Spain
}

Received April 27, 2004. In Final Form: J uly 2, 2004

\begin{abstract}
Biomolecular interactions over the surface of a microcantilever can produce its bending motion via changes of the surface stress, which is referred to nanomechanical response. Here, we have studied the interaction forces responsible for the bending motion during the formation of a self-assembled monolayer of thiolated 27-mer single-stranded DNA on the gold-coated side of a microcantilever and during the subsequent hybridization with the complementary nucleic acid. Theimmobi lization of thesingle-stranded DNA probe gives a mean surface stress of $25 \mathrm{mN} / \mathrm{m}$ and a mean bending of $23 \mathrm{~nm}$ for microcantilevers with a length and thickness of about $200 \mu \mathrm{m}$ and $0.8 \mu \mathrm{m}$, respectively. The hybridization with the complementary sequence could not be inferred from the nanomechanical response. The nanomechanical response was compared with data from well-established techniques such as surface plasmon resonance and radiolabeling, to determi nethesurface coverageand study theintermol ecular forces between neighboring DNA molecules anchored to the mi crocantilever surface. From both techniques, an immobilization surface density of $3 \times 10^{12} \mathrm{~mol}$ ecules $/ \mathrm{cm}^{2}$ and a hybridization efficiency of $40 \%$ were determined. Moreimportantly, label-free hybridization was clearly detected in the same conditions with a conventional sensor based on surface plasmon resonance. The results imply that the nanomechanical signal during the immobilization process arises mainly from the covalent attachment to the gold surface, and the interchain interactions between neighboring DNA molecules are weak, producing an undetectable surface stress. We conclude that detection of nucleic acid hybridization with nanomechanical sensors requires reference cantilevers to remove nonspecific signals, more sensitive mi crocantilever geometries, and immobilization chemistries specially addressed to enhance the surface stress variations.
\end{abstract}

\section{Introduction}

Microcantilever-based sensors have recently emerged as a promising al ternativefor the detection of biomolecular interactions. Molecular recognition induces changes in the mechanical properties of the cantilever beam, which are detected via variations of the deflection or vibration. Due to the induced nanometer-scale motion of the beam during molecular recognition, the microcantilever re sponse is usually called nanomechanical response. A variety of biomolecular interactions have been detected with this technology including bacteria, proteins, pesticides, and DNA. ${ }^{1-9}$ Thepotential of this new kind of sensor

\footnotetext{
* Corresponding author. Tel: +34 91 806/3338/0700. Fax: +34 91 8060701. E-mail: jtamayo@imm.cnm.csic.es.

† Instituto de Microelectrónica de Madrid, IMM-CSIC.

₹ Centro Nacional de Biotecnología, DIO-CNB-CSIC.

(1) Raiteri, R.; Grattarola, M.; Butt, H.-J .;Skádal, P. Sens. Actuators, B 2001, 79, 115-126.

(2) Wu, G.; Datar, R.; Hansen, K.; Thundat, T.; Cote, R.; Majumdar A. Nat. Biotechnol. 2001, 19, 856-860.

(3) Álvarez, M.; Calle, A.; Tamayo, J .; Lechuga, L. M.; Abad, A.; Montoya, A. Biosens. Bioelectron. 2003, 18,649-653.

(4) Moulin, A. M.; O'shea, S.J .; Welland, M. E. Ultramicroscopy 2000,
} $82,23-31$.

(5) Fritz, J .; Baller, M. K.; Lang, H. P.; Rothuizen, H.; Vettiger, P.; Meyer, E.; Güntherodt, H.J .; Gerber, C.; Gimzewski, J . K. Science2000 288, 316-318.

(6) Wu, G.; J i, H.; Hansen, K.; Thundat, T.; Datar, R.; Cote, R.; Hagan, M. F.; Chakraborty, A. K.; Majumdar, A. Proc. Natl. Acad. Sci. U.S.A 2001, 98, 1560-1564

(7) Hansen, K.; J i, H.-F.; Wu, G.; Datar, R.; Cote, R.; Majumdar, A.; Thundat, T. Anal. Chem. 2001, 73, 1567-1571.

(8) McKendry, R.; Zhang, J .; Arntz, Y.; Strunz, T.; Hegner, M.; Lang H. P.; Baller, M. K.; Certa, U.; Meyer, E.; Güntherodt, H.-J .; Gerber, C. Proc. Natl. Acad. Sci. U.S.A. 2002, 99 (15), 9783-9788.

(9) Hagan, M. F.; Majumdar, A.; Chakraborty, A. K.J . Phys. Chem. B 2002, 106, 10163-10173. relies on the combination of direct detection without the need for labeling with fluorescent molecules, tiny sensor area of the order of $1000 \mu \mathrm{m}^{2}$, and scalability by microfabrication of cantilever arrays using standard semiconductor technology. Nanomechanical biosensors are commonly based on the beam bending method, which measures differences in the surface stress between the opposite sides of a microcantilever that are different in composition. One side of the cantilever is sensitized with the biomolecular receptor of the target molecules; hence target/receptor molecular recognition on the cantilever surface produces a differential surface stress between the opposite sides of the lever. This is energetically balanced via unequal changes of area of both surfaces that result in a cantilever bending. The relationship between the cantilever bending and the change of surface stress was discovered by $\mathrm{G}$. Stoney morethan one century ago, ${ }^{10}$ and it is given by

$$
\frac{1}{\mathrm{R}}=6 \frac{(1-v) \mathrm{L}^{2}}{\mathrm{ET}^{2}}\left(\Delta \sigma_{\mathrm{t}}-\Delta \sigma_{\mathrm{b}}\right)
$$

where $\mathrm{R}$ is the curvature radius of the cantilever, $\mathrm{L}$ and $\mathrm{T}$ are the cantilever length and thickness, $\mathrm{E}$ and $v$ are the Young's modulus and Poisson coefficient of the cantilever material, and $\Delta \sigma_{\mathrm{t}}$ and $\Delta \sigma_{\mathrm{b}}$ are the change of surfacestress of the top and bottom surfaces of the cantilever, respectively. In particular, several authors have recently reported thelabel-free detection of DNA hybridization with high sensitivity. ${ }^{5-9}$ The potential of microcantilever sen-

(10) Stoney, G. G. Proc. R. Soc. London, Ser. A 1909, 82, 172-175. 
sors for label-free detection of femtomolar amounts of biomolecules can revolutionize current techniques employed in functional genomics that requiretime-consuming sample labeling with fluorescent molecules and large amounts of RNA or DNA sample. However, the reported works show discrepancy between their results, and the origin of the nanomechanical response during molecular recognition has not been elucidated yet. I $n$ all theseworks, the cantilever is sensitized with single-stranded (ss) DNA probes that have been derivatized with a thiol linker for covalent attachment on the gold-coated side of the cantilever. Hybridization with complementary DN A produced a measurable deflection of the cantilever whose magnitude and sign have varied in different reports. 5,6,8,9 Authors have reported compressive stress during DNA hybridization, which is interpreted as theincrease of steric forces between neighboring DNA chains during hybridization. Other authors have observed compressive and tensile surface stress depending on the buffer ionic strength. ${ }^{6}$ This response is explained as a result of the interplay between electrostatic and conformational entropy forces. Moreover, only few works use reference cantilevers to disregard artifacts due to nonspecific adsorption, slight variations of buffer conditions $(\mathrm{pH}$ and ionic strength), and local temperaturechanges. 5,8,11 I $\mathrm{n}$ the present work, we study the origin of the nanomechanical response during hybridization for microcantilevers that have been sensitized with thiolated DNA probes as in the previous reports. I n contrast to previously reported works, we have found that the duplex formation gives a small cantilever bending that is similar to that obtained with noncomplementary ssDNA. This indicates that hybridization-driven surface stress is bel ow the detection limit of sensor devices working with a single microcantilever with length smal ler than $200 \mu \mathrm{m}$ and thickness above 800 $\mathrm{nm}$. To determine the forces that contribute to the cantilever bending, thenanomechanical signal during the attachment of the ssDNA probes to the gold-coated side of the microcantilever was measured and compared to the hybridization signals. In addition, both cantilever responses due to the ssDNA probe immobilization and subsequent hybridization were characterized with well-established techniques such as surface plasmon resonance, ${ }^{12-14}$ fluorescence, ${ }^{15}$ and radiolabeling. ${ }^{16}$

\section{Materials and Method}

2.1. Materials. Commercially available silicon nitride cantilevers from Olympus were used, which are $200 \mu \mathrm{m}$ long, $40 \mu \mathrm{m}$ wide, and $0.8 \mu \mathrm{m}$ thick. The nominal spring constant is 0.10 $\mathrm{N} / \mathrm{m}$. Prior to theexperiments, theoriginal reflectivegold coating of the cantilever was removed by immersion in a mixture of hydrochloric acid and nitric acid (3:1), and this cantilever side was coated with $5 \mathrm{~nm}$ of chromium and $20 \mathrm{~nm}$ of gold using thermal evaporation. The chromium layer was used to improve the adhesion between the gold and the silicon nitride. The use of freshly evaporated gold resulted in a more reproducible and a more packed monolayer of thiolated DNA. This is due to the oxidation and contamination of the gold surface with the time. Also, the gold-coated microcantilevers wereimmersed in piranha

(11) Lang, H. P.; Hegner, M.; Meyer, E.; Gerber, Ch. Nanotechnol ogy 2002, 13, R29-R36

(12) Thiel, A. J .; Frutos, A. G.; J ordan, C. E.; Corn, R. M.; Smith, L. M. Anal. Chem. 1997, 69, 4948-4956.

(13) Georgiadis, R.; Peterlinz, K. P.; Peterson, A. W. J . Am. Chem. Soc. 2000, 122, 13 .

(14) Peterson, A. W.; Wolf, L. K.; Georgiadis, R. M.J . Am. Chem. Soc 2002, 124, 14601-14607.

(15) Cosstick, R.; McLaughlin, L. W.; Eckstein, F. NucleicAcids Res. 1984, 12, 1791-1810.

(16) Chaconas, G.; Van de Sande, J. H.; Church, R. B. Biochem Biophys. Res. Commun. 1975, 66, 962-969. solution $\left(70 \% \mathrm{H}_{2} \mathrm{SO}_{4}\right.$ and $\left.30 \% \mathrm{H}_{2} \mathrm{O}_{2}\right)$ to clean the surface before each experiment.

DNA probes were 27 nucleotides in length and were synthesized with a thiol linker group, $\mathrm{SH}-\left(\mathrm{CH}_{2}\right)_{6}$, at the $5^{\prime}$ end with a nucleotide sequence given by $5^{\prime}$-TTT TTT TTT TTT TTT AAC GAC GGC CAG-3'. The 15-T tail is employed as a vertical spacer chain to increase the accessibility to the complementary 12-mer DNA target, 5'-CTG GCC GTC GTT-3'. Control experiments were performed with DNA with a noncomplementary sequence, 5'AAC GAC GGC CAG-3'. Theoligonucl eotides were commercially purchased from I ntegrated DNA Technologies. The immobilization and hybridization buffers were prepared with $50 \mathrm{mM}$ phosphate buffered (PB) solution, $\mathrm{pH} 7.0$, and 0.5 and $1 \mathrm{M} \mathrm{NaCl}$, respectively.

In some experiments, the cantilever sensitized with the thiolated DNA probes was treated with 6-mercapto-1-hexanol $(\mathrm{MCH})$ to improve accessibility of DNA probes to DNA targets and avoid DNA physisorption during hybridization. In this protocol developed by Herne and Tarlov, 17 a $1 \mathrm{mM}$ solution of $\mathrm{MCH}$ in PB buffer with $0.5 \mathrm{M} \mathrm{NaCl}$ was injected into the liquid cell after oligonucleotide immobilization. $\mathrm{MCH}$ was purchased from Sigma Chemical Co.

2.2. Radiolabeling Measurements. Scintillation counting is used to quantify the surface coverage of ${ }^{32} \mathrm{P}$-labeled oligonucleotides. Thelabel ing is performed by using T4 polynud eotide kinase (New England Biolabs) and [ $\gamma$-32P]ATP (Amersham Biosciences), with the unincorporated label removed by filtering the kinase reaction through Microbio-spin P-30 Tris chromatography columns (Biorad). The label efficiency is about $10 \%$. The radiolabeling measurements were performed in static and in flow conditions using gol d-coated slides. I n thestatic conditions, $1 \mu \mathrm{L}$ of DNA solution is deposited over a gold-coated glass slide for $1 \mathrm{~h}$ before rinsing. In flow experiments, the conditions were similar to those employed in the cantilever sensor. Thefluid cell was sealed with a gold-coated glass slide at the same flow rate as in microcantilever experiments. We found a similar DNA coverage in static and flow conditions.

2.3. Surface Plasmon Resonance Measurements. The surface plasmon resonance(SPR) measurements were performed with a double-channel SPR sensor (Sensia Ltd.), based on the Kretschmann configuration toachievetheresonant condition by total internal reflection. ${ }^{18,19}$ The device uses a polarized $3 \mathrm{~mW}$ laser diode source at $670 \mathrm{~nm}$, and it allows the simultaneously measurement in two fluid cells of $300 \mathrm{~nL}$ each, one of them being used as a reference signal. The sensing is based on variations of the refractive index of the medium adjacent to the metal sensor surface (gold in this case). Measurements are carried out at a fixed angle of incident light in order to monitor changes in the refractive index as variations in the reflected intensity.

2.4. Microcantilever Sensor. The cantilever deflection measurements werecarried out with a home-built sensor, based on thewell-known optical beam deflection method. A laser beam is focused on the free end of the cantilever, and the deflection of the reflected beam is measured by picking up the reflected light into a four-segmented photodetector. The deflection detection limit is about $0.1 \mathrm{~nm}$. The photocurrents of the upper and lower segments are amplified and connected to an analog-to-digital data acquisition card (National Instruments) for deflection monitoring in real time. Acquisition software was programmed in Labview (National Instruments).

The experiments were carried out in a commercial fluid cell with an enclosed volume of about $50 \mu \mathrm{L}$ (Digital Instruments, Veeco), with an inlet and an outlet for liquid flow. The top part of the fluid cell is madeout of glass, and the bottom is closed with a coverslip using a rubber O-ring. The system is isolated from mechanical vibrations. The temperature could be fixed and kept constant by appropiate control of theel ectrical current of a Peltier cell placed in contact with the coverslip. F low and injection of the sample are performed by using a peristaltic pump (Gilson). The flow ratein theseexperiments was $0.3 \mu \mathrm{L} / \mathrm{s}$, approximately. Before each experiment, the cantilever is equilibrated under flow of the

(17) Herne, T. M.; Tarlov, M. J . J . Am. Chem. Soc. 1997, 119, 38 60.

(19) Aguilar, M. R.; Gallardo, A.; Lechuga, L. M.; Calle, A.; San Romn, J. Macromol. Biosci., in press. 


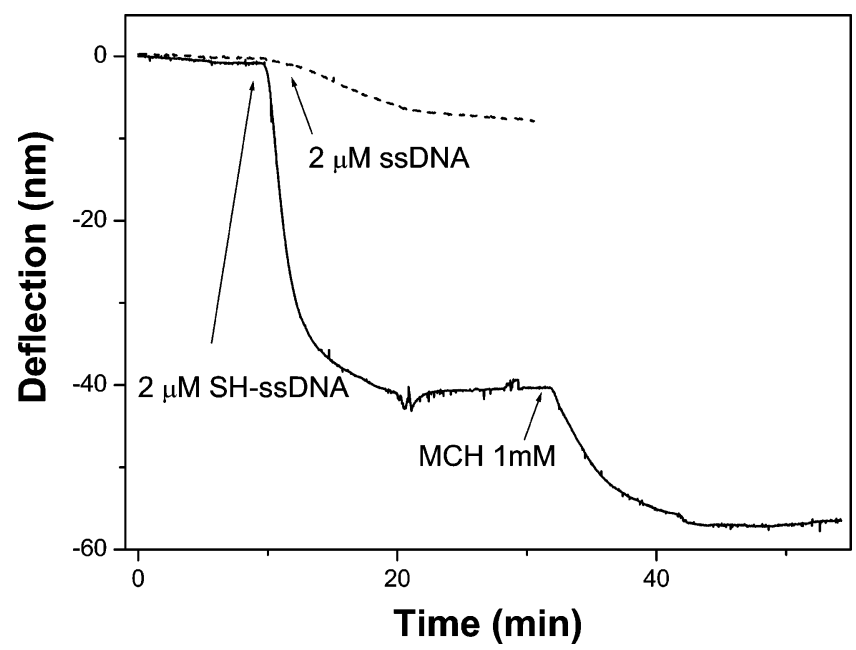

Figure 1. Cantilever deflection as function of time for a cantil ever exposed to thenonmodified DNA probe(dashed line), a cantilever exposed to a thiolated DNA probe, and subsequent adsorption of $\mathrm{MCH}$ (solid line).

buffer solution to decreasethe drift effect over themeasurements, until a baseline is obtained.

\section{Results and Discussion}

3.1. Immobilization. The nanomechanical response of a gold-coated cantilever was monitored in real time during the immobilization of the ssDNA probes. Figure 1 shows the comparison between the nanomechananical responses when the cantilever is exposed to the 27-mer DNA probe with and without modification with the thiol linker, $\mathrm{SH}-\left(\mathrm{CH}_{2}\right)_{6}-$. Both DNA probes produce a downward cantilever deflection; however thiolated DNA gives a higher and faster bending. In fact, thiol-modified ol igonucleotides spontaneously form self-assembled monolayers on gold surfaces through the coval ent bond between the gol $d$ and the sulfur atom of the thiol linker. The time constant of theadsorption was determined by fitting both curves with the L angmuir adsorption isotherm model, for which $\theta \propto 1-\exp (-\kappa \mathrm{t})$, where $\theta$ is the coverage, $\mathrm{t}$ is the time, and $\kappa$ is the reaction rate constant. ${ }^{20}$ The fittings gavetimeconstants of about $5 \mathrm{~min}$ (observed rateconstant $\left.\kappa=0.2 \mathrm{~min}^{-1}\right)$ and $1.6 \mathrm{~min}\left(\kappa=0.6 \mathrm{~min}^{-1}\right)$ for the nonmodified and modified oligonucleotides, respectively. The downward bending implies compressive surface stress; that is, the gold-coated side expands with respect to the silicon nitride backside.

Figure 2 shows an histogram of the surface stress produced by the immobilization of the thiol-modified SSDNA in PB buffer with $0.5 \mathrm{M} \mathrm{NaCl}$. For the concentrations used in the statistic, $2-5 \mu \mathrm{M}$, a mean value of 25.6 $\mathrm{mN} / \mathrm{m}$ was obtained. The histogram peak broadening is attributed to uncertainties of the mechanical properties of the cantilevers and of the gold surface. For instance, in the same fabrication process, the cantilevers exhibit small variations of thethickness and of the silicon nitride stoichiometry that produce deviations in the response to surface stress changes. On the other hand, variations in the roughness and oxidation of the gold surface can al so produce different cantilever responses. Interestingly, similar bending results areobtained during theadsorption of thethiolated probes when the concentration of the $\mathrm{NaCl}$ of the PB buffer was varied from 0 to $1 \mathrm{M}$. I n addition, no differences with the salt concentration were found in the DNA surface coverage by fluorescence microscopy using fluorescent-label ed oligonucl eotide probes.

The cantilever deflection response is proportional to thesurfacestress difference between oppositesides of the

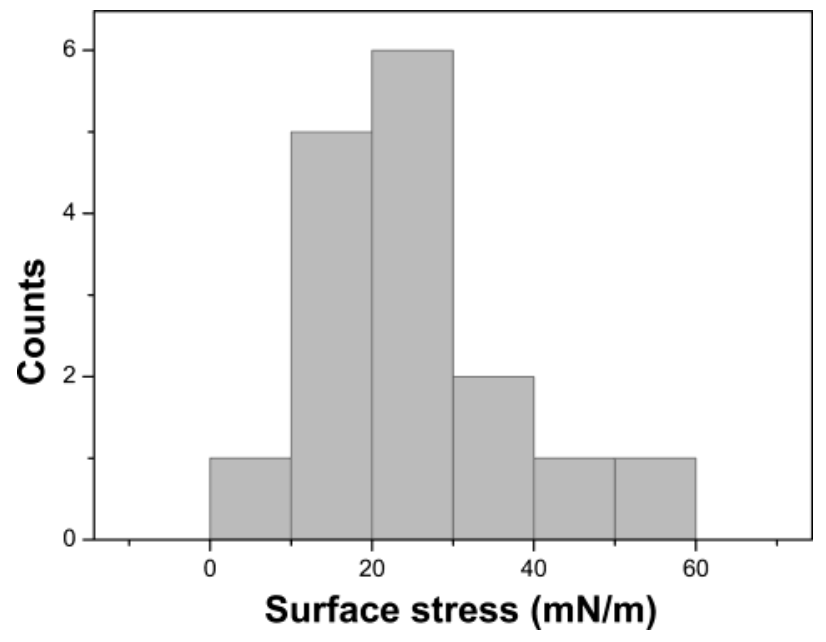

Figure 2. Histogram of the surface stress induced by the immobilization of the thiol-modified ssDNA.

cantilever. To decouple the contributions of the gold and silicon nitridesurfaces of thecantilever, theradiolabeling technique was applied to estimate the surface coverage, and the contribution of the silicon nitride backside was determined by passivating the gold-coated surface with thehydroxyl-ended alkylthiol MCH. This moleculeforms a denseself-assembled monolayer on the gol d via covalent bonding between the gold and thiol group of the $\mathrm{MCH}$. The opposite terminal hydroxyl group $(-\mathrm{OH})$ exhibits negligible interaction with the DNA, inhibiting its physisorption. The response of the passivated cantilever to thenonmodified and thiol-modified DNA gavea negl igible nanomechanical response (data not shown). The radiolabeling characterization showed a coverage signal of the P32-label ed thiolated DNA probebelow the detection limit on the passivated gold surface, while the coverage of the silicon nitride backside was $\sim 10^{11}$ molecules $/ \mathrm{cm}^{2}$. There fore, the silicon nitride surface does not significantly contribute to the nanomechanical response.

To estimate the density of the DNA grafted to the gold and understand theorigin of thesurfacestress during the DNA adsorption on the cantilever, the radiolabeling technique was applied. Surprisingly, both DNA probes, nonmodified and thiol-modified DNA, attach to the gold with a similar surface density of about $2 \times 10^{12}$ and $3 \times$ $10^{12}$ molecules $/ \mathrm{cm}^{2}$, respectively. Physisorption of the nonmodified ssDNA is related to multipleadsorptivesites between theaminegroups of thenucleotidechain and the gold. This indicates that the nanomechanical response is not strictly governed by the number of adsorbed DNA molecules, and it critically depends on the nature of the interaction between the gold and the DNA. From these results, we deduce that the nucleotide chain interaction with the gold induces smaller surface stress than the covalent bond between the sulfur and the gold.

Because of the attractive interaction between the nucleotidechain and thegold, themost favorablestructure for adsorbed thi olated DNA monolayer is theDNA strongly anchored at the surface by the thiol linker, with the nucleotide chain in contact with the surface. To enhance the accessibility of the DNA probes to the target, the surface was exposed to $\mathrm{MCH}$ after DNA immobilization. In this treatment developed by Herne and Tarlov, ${ }^{17}$ the thiol group of $\mathrm{MCH}$ rapidly displaces the weaker adsorptive contacts between the nucleotide chain and gold, covalently attaching to the interstitial regions between ssDNA chains. The post-treatment ensures that the DNA probes are only attached to the gold surface through the 


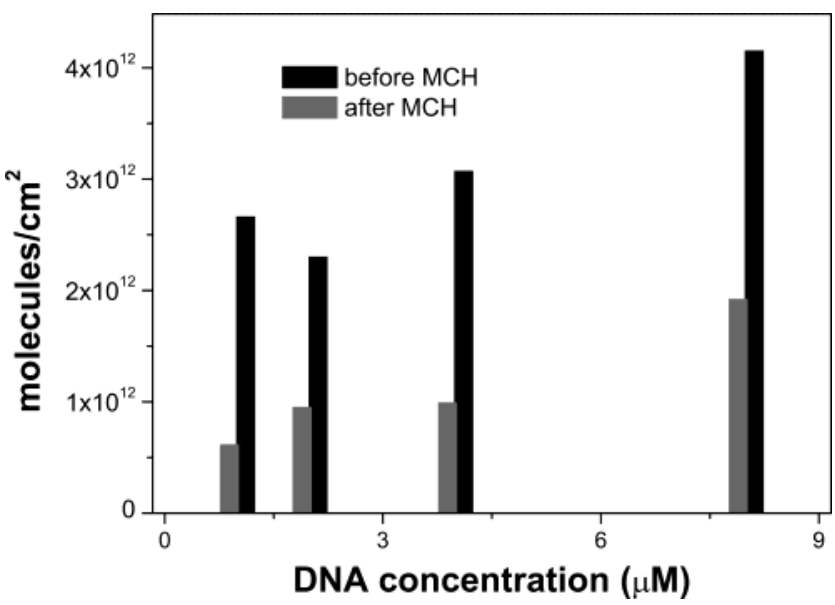

Figure 3. Radiolabeling measurement of the thiolated DNA coverage on gold before and after the $\mathrm{MCH}$ treatment.

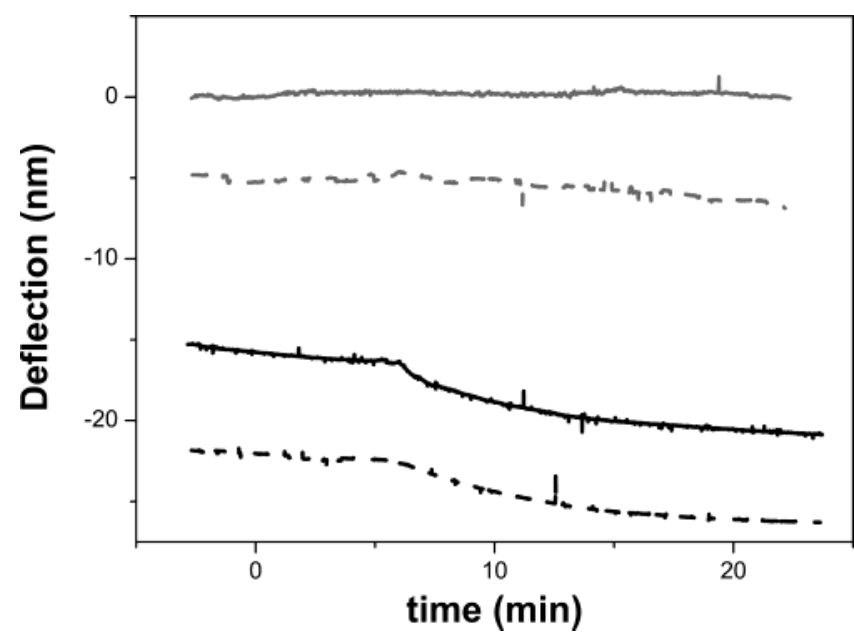

Figure 4. Deflection as a function of time after the injection of the complementary (solid lines) and noncomplementary (dashed lines) DNA, for cantilevers sensitized with thiolated DNA, with (gray lines) and without $\mathrm{MCH}$ treatment (black lines).

terminal sulfur atom of the thiol linker as the DNA negligibly interacts with thehydroxyl end of $\mathrm{MCH}$. It has been reported that $\mathrm{MCH}$ post-treatment produces an increase of thehybridization efficiency fromless than $10 \%$ to $80 \%$ approximately. ${ }^{17,21}$

Theadsorption of $\mathrm{MCH}$ after thiolated DNA attachment was monitored in real time (Figure 1 ). $\mathrm{MCH}$ adsorption produces a large compressive surface stress, giving a downward bending of about $30-35 \%$ of the cantilever deflection resulting from the thiolated DNA immobilization. Additionally, radiolabeling experiments were performed to characterize the MCH treatment (Figure 3). The results indicate that the $\mathrm{MCH}$ molecules displace about $60 \%$ of theimmobi lized DNA, suggesting that only $40 \%$ of the immobilized chains are coval ently attached to the gold surface.

3.2. Hybridization. Figure 4 shows four curves corresponding with the evolution of the cantilever deflection when a complementary and noncomplementary ssDNA is flowed over cantilevers sensitized with the thiolated DNA probe, with and without MCH post-treatment. The target employed is a 12-mer oligonucleotide complemen-

(20) Marie, R.; J ensenius, H.; Thaysen, J .; Christensen, C. B.; Boisen, A. Ultramicroscopy 2002, 91, 29-36.

(21) Steel, A. B.; Levicky, R. L.; Herne, T. M.; Tarlov, M. J . Biophys. J . 2000, 79, 975-981. tary to the 12 bases of the DNA probe more separated from the thiol linker. The previous functionalization of the cantilever was performed under flow and monitored in real timeas shown in the previous section, for checking the immobilization. For the cantilever without $\mathrm{MCH}$ treatment, the injection of both complementary and noncomplementary DNA produced a downward bending (compressive stress) of about $10 \%$ of the immobilizationinduced deflection. A reproducible difference in the bending duetothecomplementary and noncomplementary DNA was not observed. A lower cantilever bending for the complementary and noncomplementary ssDNA was found when the cantilever was treated with $\mathrm{MCH}$ to enhance the accessibility of the DNA probe and reduce the physisorption. The lower bending is related to the lower physisorption duetothe $\mathrm{MCH}$ treatment. However, as it occurs with the cantilever without $\mathrm{MCH}$ treatment, no significant and reproducible difference was found between the cantilever response to the control and complementary DNA.

The hybridization on the cantilevers was checked ex situ by using fluorescence microscopy and labeling of the complementary and noncomplementary DNA target with the fluorescent dye Cy5. Significant fluorescence was observed on thegold-coated side of thecantilever with the complementary DNA, while the control signal was negligible. This is in contrast with the cantilever responses obtained here. Figure 5shows thestatistic of thecantilever bendings for more than 60 experiments where no significant difference was found between the cantilever responses due to the complementary and noncomplementary DNA (Figure 5). The average cantilever bending is about $10 \%$ of that obtained during immobilization of the thiolated DNA probe.

To understand the nanomechanical response of the sensor during hybridization, SPR experiments were performed. SPR is a well-established optical technique that allows the measurement of the adsorption coverage on gold surfaces based on the refractive index of the adsorbates. The SPR signal can be directly compared to the nanomechanical signal as both sensors use a gold sensing surface and labeling is not required. However, while the SPR signal is proportional to the mass surface density, the relationship between the cantilever bending and the coverage is not straightforward, and it has not been elucidated yet. Figure 6 shows the SPR signal as a function of timeduring theimmobilization and subsequent hybridization. A MCH treatment was applied, though similar results were obtained without $\mathrm{MCH}$ treatment. A significant and fast hybridization signal was obtained with the complementary DNA, while the noncomplementary DNA produced a negligibleresponse. Moreover, thesurface could be regenerated several times by dissociating the duplex with $0.2 \mathrm{M} \mathrm{NaOH}$, preserving the hybridization efficiency. F rom the SPR signals, a hybridization yield of about $40 \%$ was determined.

3.3. Discussion. To understand the nanomechanical response, it is convenient to split the surface stress into twoterms: oneis dueto theinteraction between the DNA and the gold surface, and the other arises from the interactions between neighboring DNA molecules. The forces intervening in thesecond term of the surfacestress can besplit into (i) thereduction of conformational entropy of the DNA when it is anchored to the surface and neighboring molecules limit the thermal motion, (ii) the electrostatic double layer repulsion arising from the osmotic pressure between the counterions that surround the negatively charged DNA molecules, and (iii) the repulsivehydration forces resulting from the perturbation 
$\mathrm{NON} \mathrm{MCH}$
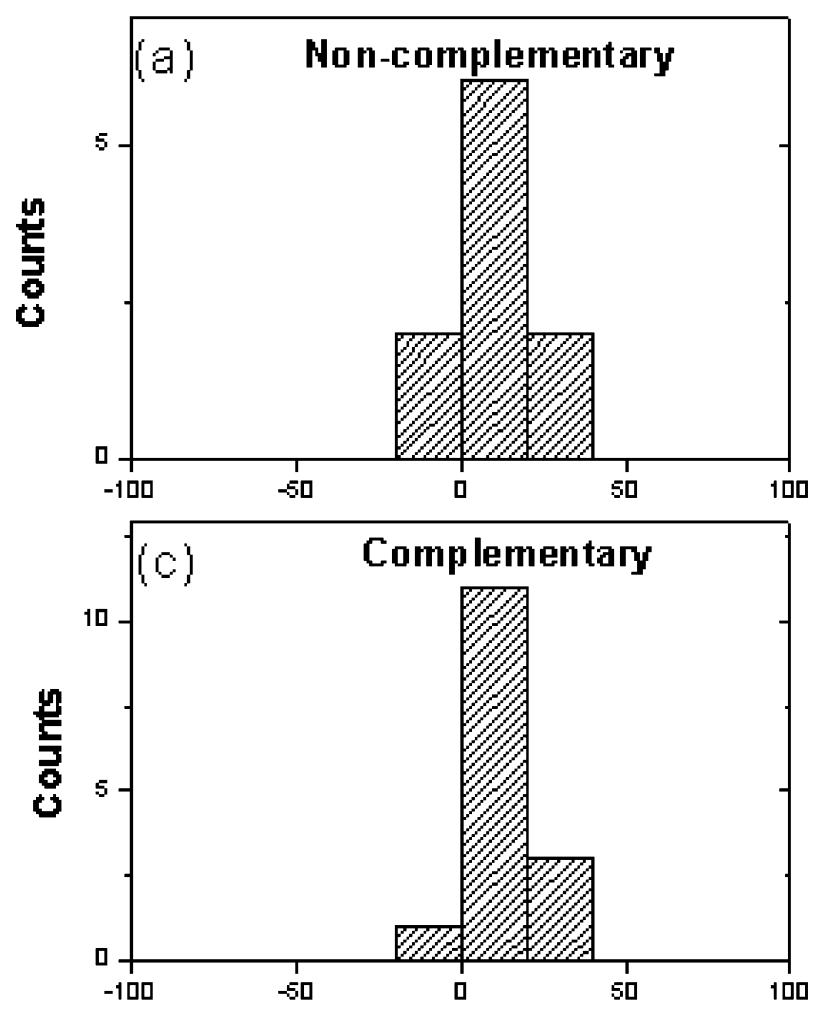

Hybridization/Immobilization B ending ( $\%$ )
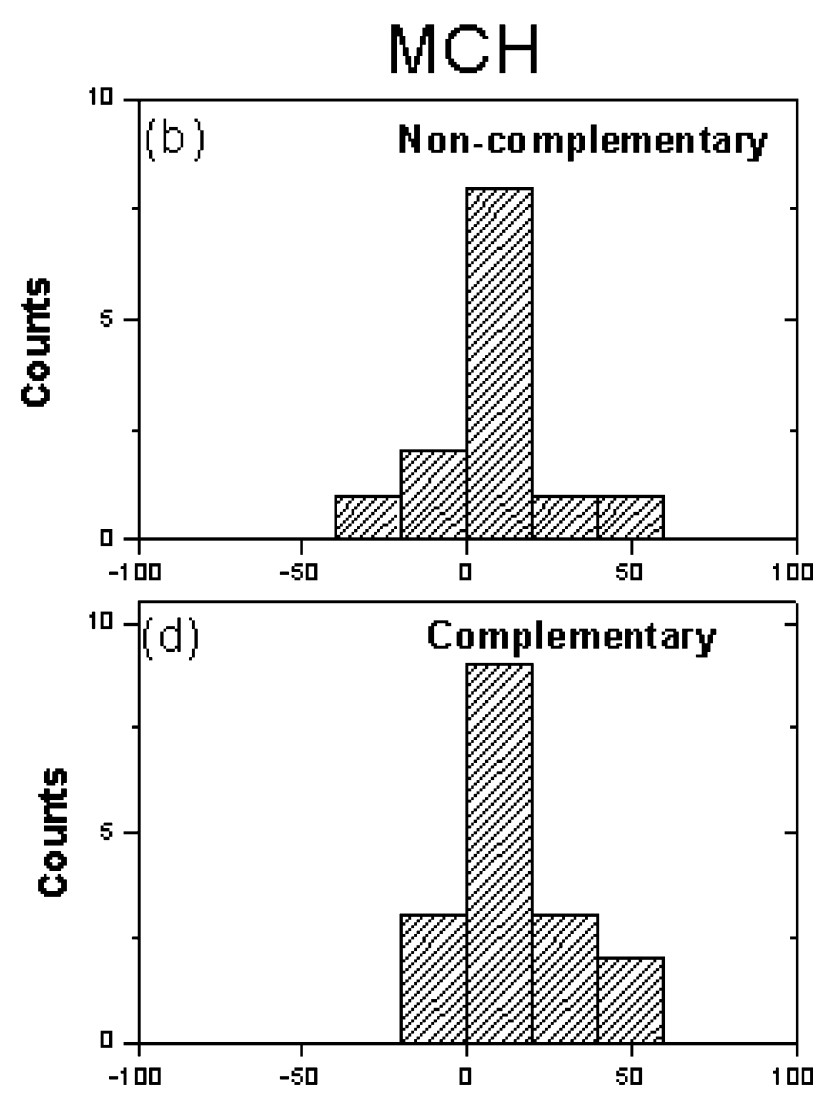

Hybridization'lmmobilization B ending (\%)

Figure 5. Statistic of the cantilever bending with respect to that due to the immobilization of the thiolated DNA probe for the injection of the noncomplementary $(a, b)$ and complementary DNA $(c, d)$, with $(b, d)$ and without $(a, c)$ MCH treatment of thesensitized cantilever surface.

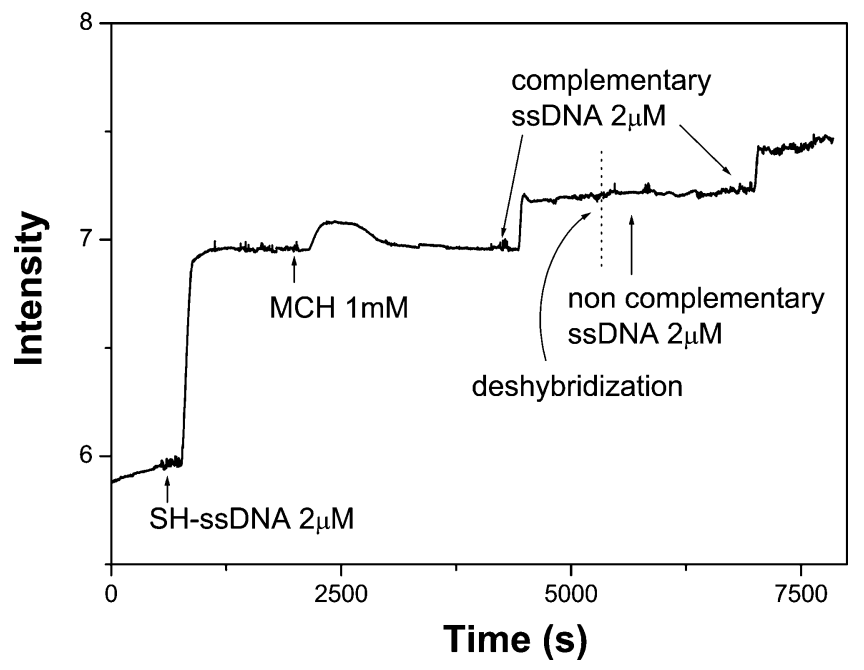

Figure 6. SPR real-time measurement of a hybridization experiment. Thesequence fol lowed in this experiment was first theimmobilization of a thiol-modified 25-mer SSDNA, treatment with $\mathrm{MCH}$, injection of a complementary 12-mer DNA, dehybridization with $0.2 \mathrm{M} \mathrm{NaOH}$, injection of the noncomplementary 12-mer DNA, and finally injection again of the complementary DNA. Immobilization and $\mathrm{MCH}$ treatment were performed in PB buffer with $0.5 \mathrm{M} \mathrm{NaCl}$, and hybridization experiments were performed in PB buffer with $1 \mathrm{M} \mathrm{NaCl}$. The experiment was performed at $24^{\circ} \mathrm{C}$.

of the hydrogen network in water by the DNA molecules. ${ }^{9,22,23}$

(22) I sraelachvili, J . Intermolecular and Surface Forces, 2nd ed.; Academic Press: London, 1991.
During the covalent attachment of the thiolated DNA probes to the gold-coated side, a partial electron transfer of the gold (donor) to the sulfur atom (acceptor) of the thiol linker is produced. The reduction of the electron density of the gold surface is alleviated by increasing the interatomic distance, resulting in a compressive surface stress. On the other hand, the three interaction forces mentioned above also contribute with a compressive surface stress as the interaction energy is relaxed by an expansion of thesensitized cantilever side. Theimportance of these repulsive interactions depends on the DNA grafting density.

From the radiolabeling and surface plasmon resonance data, an average distance between the surface-anchored thiolated DNA probes of about $6 \mathrm{~nm}$ is determined. This is in contrast with the interchain distance of the thiol linker without DNA that is about $\sim 0.5 \mathrm{~nm}$. It seems that the large size of the DNA molecule compared to that of thethiol linker produces a drasticreduction of the surface density due to the steric hindrance. To determine the contribution of the conformational entropy forces, it is useful to compare the size of the DNA molecule free in solution with the average distance between neighboring DNA molecules on the gold surface. In solution, the DNA assumes the shape of a random coil, with a characteristic size given by the radius of gyration $\mathrm{Rg}$, which represents theroot-mean-square radius of the polymer coil in solution. Rg was estimated by Tinland et al. ${ }^{24}$ as $\mathrm{Rg}=(0.43 \mathrm{~N} \rho / 3)^{1 / 2}$ $\mathrm{nm}$, where $\mathrm{N}$ is the base number and $\rho$ is the SSDNA

(23) Levicky, R.; Herne, T. M.; Tarlov, M. J .; Satija, S. K. J . Am. Chem. Soc. 1998, 120, 9787-9792.

(24) Tinland, B.; Pluen, A.; Sturm, J .; Weil, G. Macromol ecules 1997, 30, 5763-5765. 
persistence length, which represents the distance al ong the chain backbone over which a strand of SSDNA will behave like a rigid rod. For the high salt concentration limit, $\rho \simeq 0.75 \mathrm{~nm}$ and the 27-mer ssDNA probe has a gyration diameter, $2 \mathrm{Rg}$, of about $3.4 \mathrm{~nm}$, which is significantly smaller than the interneighboring distance of $6 \mathrm{~nm}$. This indicates that the thermal motion is hardly perturbed by the presence of neighbors, and the contribution of the conformational entropy force to the surface stress should be small. On the other hand, the Debye length in the immobilization buffer was $\lambda^{-1}=0.43 \mathrm{~nm}$; hence the contribution of the el ectrostatic force should be negl igible, as it approximately decays with theexponential of the ratio between the distance and Debye length. In fact, we have not found an ionic-strength dependence of the cantilever response due to the DNA immobilization. Finally, hydration forces exponentially decay with a characteristic length of about 0.3 , which is again much smaller than the average distance.

Thesamerationaleused abovecan beapplied toanalyze the intermolecular forces that arise during hybridization. During hybridization, the DNA duplex chain adopts a stiffer rodlike structure due to the higher persistence length of $50-80 \mathrm{~nm}$. Thus a diminution of the configurational entropy is expected that would result in a tensile surfacestress. ${ }^{6,9}$ However, this effect is small at the surface density of DNA probes achieved here. On the other hand, the formation of the double hel ix gives rise to an increase of the negative charges. However, the increase of the electrostatic repulsion forces should not produce a noticeablesurfacestress as thehybridization buffer has a Debye length of $\lambda^{-1}=0.3 \mathrm{~nm}$. Finally, as occursin theimmobilized single-stranded DNA, a significant contribution of the hydration force is not expected during hybridization.

We conclude that the main source of surface stress during the immobilization is the covalent bond between the surface gold atoms and the sulfur atoms of the thiol linker of the DNA probes, with a small contribution from the weak interactions between the nucleotide chain and the gold. In contrast, the only contribution to the surface stress during hybridization is the intermolecular forces between neighboring DNA molecules. However, for the interneighboring distance of about $6 \mathrm{~nm}$ obtained here, this contribution is very small, and the generated surface stress should be below the detection limit of the sensor used here. Theoptical beam deflection techniqueemployed in the sensor has an accuracy of $0.1 \mathrm{~nm}$. However, variations of the temperature $\left( \pm 0.1^{\circ} \mathrm{C}\right)$ and slight variations of the electrolyte concentration produce significant deflections of $2-5 \mathrm{~nm}$ due to the bimetallic effect. This could hide the surface stress generated during the DNA duplex formation that is about $1 \mathrm{mN} / \mathrm{m}$ based on reported experiments with arrays of microcantilevers. ${ }^{5,8}$ This should produce a deflection of about $1 \mathrm{~nm}$ in the cantilevers used here.

Therefore, the detection of nucleic acid hybridization with nanomechanical sensors needs reference cantilevers sensitized with noncomplementary DNA to decouple the molecular recognition signal from nonspecific signals. It is also interesting to notice that the detection of other kind of biomolecules is not so demanding. For instance, we havesuccessfully applied nanomechanical sensors for detection of pesticides in the nanomolar range using antigen/antibody recognition and using the same sensor device. ${ }^{3}$ This highlights theneed for a major understanding of the mechanisms responsible for surface stress due to the biomolecular interactions. This knowledge is crucial for the devel opment of immobilization procedures in which the geometry of the receptor molecules is addressed to generate high interaction forces between neighboring molecules during molecular recognition.

Acknowledgment. This work was supported by the MyCT (BIO2001-1235-C03-01).

LA0489559 